\title{
Fast Response and Temporal Coherent Oscillations in Small-World Networks
}

\author{
Luis F. Lago-Fernández, ${ }^{1}$ Ramón Huerta, ${ }^{1,2}$ Fernando Corbacho, ${ }^{1}$ and Juan A. Sigüenza ${ }^{1}$ \\ ${ }^{1}$ Grupo de Neurocomputación Biológica (GNB), E.T.S. de Ingeniería Informática, Universidad Autónoma de Madrid, \\ 28049 Madrid, Spain \\ ${ }^{2}$ Institute for Nonlinear Science, University of California, San Diego, La Jolla, California 92093-0402
}

(Received 26 July 1999)

\begin{abstract}
We have investigated the role that different connectivity regimes play in the dynamics of a network of Hodgkin-Huxley neurons by computer simulations. The different connectivity topologies exhibit the following features: random topologies give rise to fast system response yet are unable to produce coherent oscillations in the average activity of the network; on the other hand, regular topologies give rise to coherent oscillations, but in a temporal scale that is not in accordance with fast signal processing. Finally, small-world topologies, which fall between random and regular ones, take advantage of the best features of both, giving rise to fast system response with coherent oscillations.
\end{abstract}

PACS numbers: 87.18.Sn, 05.45.-a, 84.35.+i, 87.18.Bb

In a recent Letter by Watts and Strogatz [1] it was shown that small-world (SW) networks enhance signalpropagation speed, computational power, and synchronizability. SW stands for a network whose connectivity topology is placed somewhere between a regular and a completely random connectivity. The main properties of these specific networks are that they can be highly clustered like regular networks and, at the same time, have small path lengths like random ones. Therefore, SW networks may have properties given neither in regular nor in random networks [2]. In this Letter we have extended Watts and Strogatz's general framework by introducing dynamical elements in the network nodes. Our source of inspiration is based on a phenomenon observed in the olfactory antennal lobe (AL) of the locust [3]. The AL is a group of around 800 neurons whose functional role is to relay information from the olfactory receptors to higher areas of the brain for further processing. Two main features have been observed in the dynamics of the AL. First, there is a fast response of the AL when the stimulus is presented. Second, when an odor is presented to the insect, coherent oscillations of $20 \mathrm{~Hz}$ in the local field potential (LFP) are measured [3]. Summarizing, fast coherent oscillations are observed. There are also other systems in the brain that present coherent LFP oscillations, hence, hinting to the generality of these phenomena (see $[4,5]$ ).

The cooperative behavior of large assemblies of dynamical elements has been the subject of many investigations $[6-10]$. In all of them the connectivity between the elements of the network was either regular (local or global all-to-all) or random. However, none of these studies incorporates a comparative analysis of network dynamics for all the different connectivity topologies.

In the present work we want to show that in order to provide fast response and coherent oscillations a SW topology is required. Without the coherent oscillations the AL seems to lose its ability to process the information incoming from the sensors [3]. The model we propose for this study is made of an array of nonidentical Hodgkin-Huxley elements coupled by excitatory synapses. The unit dynamics is described by the following set of coupled ordinary differential equations:

$$
\begin{gathered}
C_{m} \dot{V}_{i}=I^{e}-g_{L} \hat{V}_{L}-g_{\mathrm{Na}} m^{3} h \hat{V}_{\mathrm{Na}}-g_{\mathrm{K}} n^{4} \hat{V}_{\mathrm{K}}+I^{s}(t), \\
\dot{m}=\alpha_{m}(V)(1-m)-\beta_{m}(V) m \\
\dot{h}=\alpha_{h}(V)(1-h)-\beta_{h}(V) h \\
\dot{n}=\alpha_{n}(V)(1-n)-\beta_{n}(V) n
\end{gathered}
$$

where $V_{i}$ represents the membrane potential of unit $i$; $C_{m}$ is the membrane capacitance per unit area; $I^{e}(t)$ is the external current, which occurs as a pulse of amplitude $I_{0} ; I^{s}(t)$ is the synaptic current; $\hat{V}_{r}=V_{i}-V_{r}$, where $V_{r}$ are the equilibrium potentials for the different ionic contributions $(r=\mathrm{Na}, \mathrm{K})$ and passive channel $(r=L)$, and $g_{r}$ are the corresponding maximum conductances per unit area; $h, m, n$ are the voltage dependent activating and inactivating variables; and $\alpha, \beta$ are functions of $V$ adjusted to physiological data by voltage clamp techniques. We have used the original functions and parameters employed by [11]. The model neuron is sitting below the bifurcation point (quiescent state) until the input arrives that forces the system to undergo a saddle-node bifurcation on a limit cicle [12]. The system was integrated using the RungeKutta 6(5) scheme with variable time step based on [13]. The synaptic current $I^{S}$ is given by

$$
I_{i}^{s}(t)=g_{i j} r_{j}(t)\left[V_{s}(t)-E_{s}\right],
$$

where $i$ stands for the postsynaptic and $j$ for the presynaptic neuron, and $g_{i j}$ is the maximum conductance, which determines the degree of coupling between the two connected neurons. $V_{s}$ is the postsynaptic potential, $E_{s}$ is the synaptic reversal potential, and $r_{j}(t)$ is the fraction of bound receptors [14] given by the equation $\dot{r}=\alpha(t)(1-r)-\beta r$. The rise and decay constants $\alpha(t)$ and $\beta$ are given by 
$\alpha(t)=0.94 \theta\left(t_{0}+\tau-t\right) \theta\left(t-t_{0}\right) \mathrm{msec}^{-1}$ and $\beta=$ $0.18 \mathrm{msec}^{-1}$, where $\theta(x)$ is the Heaviside function, $t_{0}$ is the time when the presynaptic neuron fires (membrane potential over $27 \mathrm{mV}$ ), and $\tau=1.5 \mathrm{msec}$.

In this model three different kinds of connectivity patterns have been tested: regular, random, and small world. To interpolate between regular and random networks we follow the procedure described in [1] which we summarize here for convenience: we start from a ring lattice with $N$ vertices and $k$ edges per vertex, and each edge is rewired at random with probability $p$. The limits of regularity and randomness are for $p=0$ and $p=1$, respectively, and the SW topology lies somewhere in the intermediate region $0<p<1$. The quantification of the structural properties of these graphs is performed using the characteristic path length $L(p)$ and the clustering coefficient $C(p) . L(p)$ is defined as the number of edges in the shortest path between two vertices, averaged over all pairs of vertices. $C(p)$ is defined as follows: suppose that a vertex $v$ has $k_{v}$ neighbors; then at most $k_{v}\left(k_{v}-1\right) / 2$ edges can exist between them. Let $C_{v}$ denote the fraction of these allowable edges that actually exist, and define $C$ as the average of $C_{v}$ over all vertices $v$. Figure 1a replicates that of [1] for ease of reference.

Next we investigate the functional significance of SW topologies for the dynamics of the network. To study the global behavior of the network we compute its average activity $\overline{V(t)}=(1 / N) \sum_{i=1}^{N} V_{i}(t)$. The quantities used to

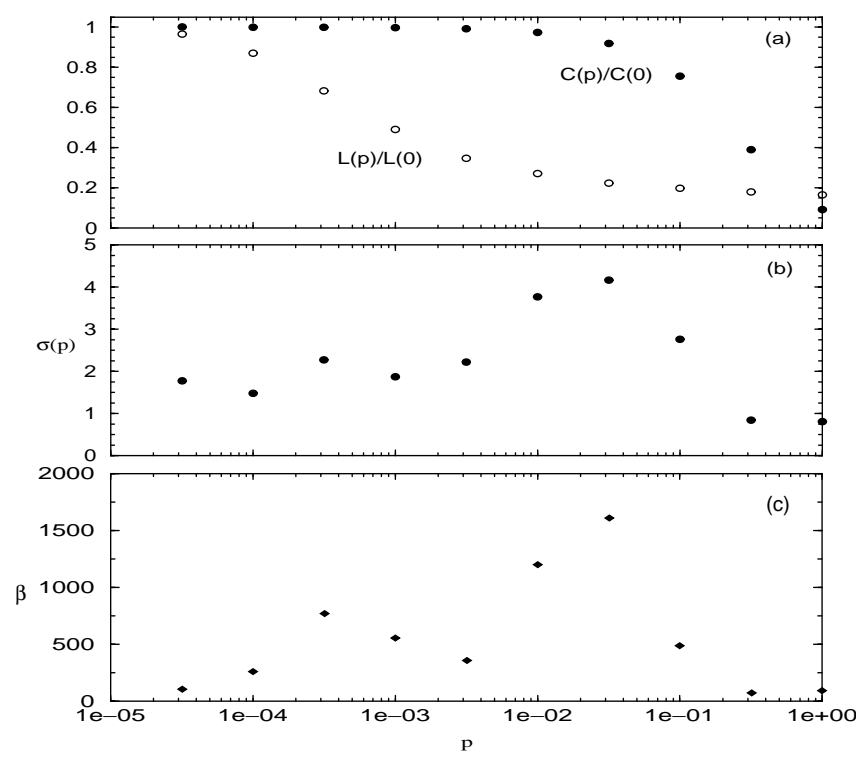

FIG. 1. (a) Characteristic path length $L(p)$ and clustering coefficient $C(p)$ for the family of randomly rewired graphs, normalized to the values $L(0)$ and $C(0)$ of the regular case. (b) Average activity oscillation amplitude $\sigma(p)$, and (c) degree of coherence $\beta(p)$ for the whole range of networks, calculated between $T_{1}=100$ and $T_{2}=200$. All curves are averages over ten realizations of the simulation with parameters $N=797$, $k=30$, and $g=0.015$. An input signal $I_{0}=1.5$ was injected, at $t=50$, to 80 contiguous neurons (10\% of the total). detect the onset and degree of coherent oscillations are the average activity oscillation amplitude [10] and the degree of coherence [15]. The amplitude of the oscillations is measured by

$$
\sigma^{2}(p)=\frac{1}{T_{2}-T_{1}} \int_{T_{1}}^{T_{2}}\left[\left\langle\overline{V_{p}(t)}\right\rangle_{t}-\overline{V_{p}(t)}\right]^{2} d t,
$$

where $\overline{V_{p}(t)}$ is the average activity of the network for a given value of the probability $p$, and the angle brackets denote temporal average over the integration interval. A high value of $\sigma(p)$ would imply a high amplitude of the oscillations of the average activity, while a low value would indicate an almost nonoscillatory behavior. The degree of coherence is determined by fitting a Gaussian to the highest peak of the power spectra and calculating

$$
\beta=H \omega / \Delta \omega,
$$

where $H$ is the height of the peak, $\omega$ is the frequency at which it appears, and $\Delta \omega$ is the width of the peak at the half maximum height [15].

In Fig. 1b we plot $\sigma(p)$ for each of the different networks characterized by its probability $p$, and in Fig. 1c we do the same for $\beta(p)$. Notice that coherent oscillations increase in the region in which a high $C(p)$ and a low $L(p)$ occur simultaneously; this is precisely the $\mathrm{SW}$ region. This can be better observed in Fig. 2, which shows the average activity of the network and the power spectra (inset) in three cases corresponding to the three different topological configurations: regular, random, and SW. Both the regular and the SW topologies display coherent oscillations, but in the regular network they appear much later and their amplitude is smaller than in the SW case. On the other hand, the random network displays only irregular variations over an

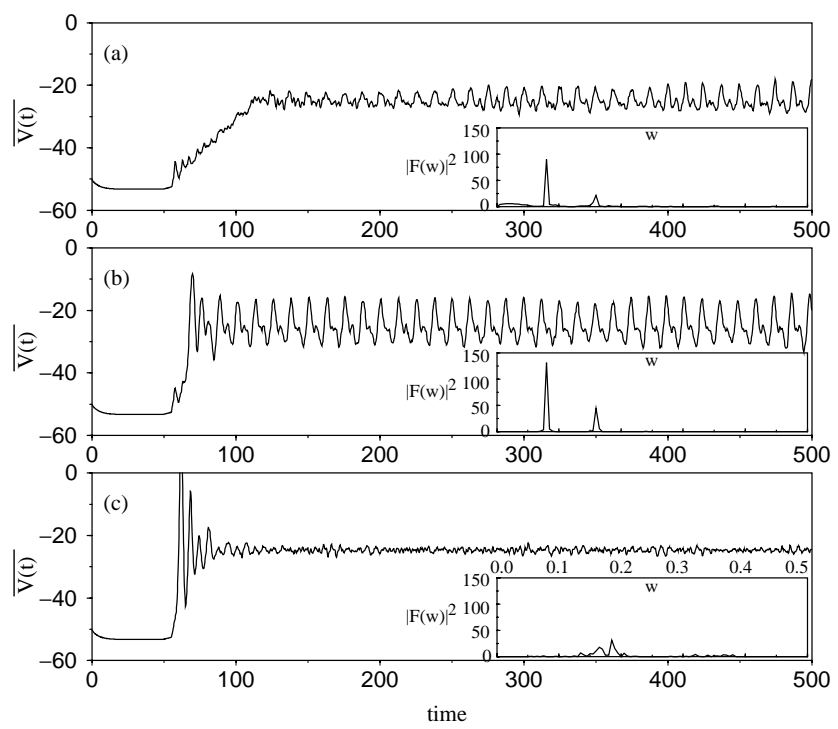

FIG. 2. Average activity and power spectrum (inset) in a network of 797 neurons. (a) Regular network $(p=0)$. (b) Smallworld network $(p=0.032)$. (c) Random network $(p=1)$. The input onset occurs at $t=50$ and is offset at $t=350$. 
almost constant pattern of activity. A more extensive study in the $(k, p)$ plane has been performed in order to establish the limits for the appearance of coherent oscillations and to check that our previous results can be generalized within a certain range of parameters. We have computed the average activity oscillation amplitude for a total of 180 points in the $(k, p)$ plane. An interpolation of these results is plotted in Fig. 3, where the clear zones indicate high values of $\sigma$. We can conclude from this figure that fast coherent oscillations appear only in the region of intermediate probabilities, that is, the SW. The a priori limits on $k$ are based on the fact that for $k$ lower than $\sim 10$ the activation of the network is very weak, while for $k$ higher than $\sim 35$ some neurons become saturated.

Temporal coding in the AL is represented by the timing of action potentials with respect to an ongoing coherent collective oscillatory pattern of activity. When an odor is presented, some neurons in the AL respond to the odor with some particular timing with respect to the LFP [3]. As a measure of this temporal coding, we have divided time in periods of the global average activity and calculated for each period the quantity

$$
A_{i}(n)=\frac{1}{C} \int_{T}\left[a_{i}(t)-\overline{V(t)}\right]^{2} d t,
$$

where $i$ represents a particular cluster (all the neurons that are directly connected to neuron $i), n$ represents a particular period of the mean activity $\overline{V(t)}$ of the whole network, $a_{i}(t)$ is the mean activity of cluster $i$, and $C$ is a normalization constant to get the value of $A_{i}(n)$ in the range $0-1$. In Fig. 4 we show the results for three different clusters chosen at random in a network within the SW connectivity regime. It can be observed that the activities of the different clusters are out of phase and reach their maximum

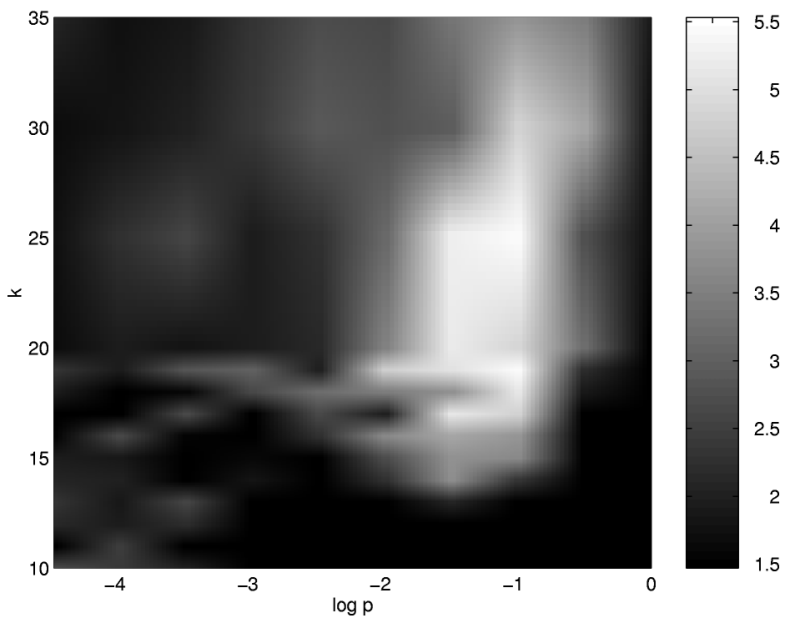

FIG. 3. Phase diagram which shows the regions of oscillatory (clear, high $\sigma$ ) and nonoscillatory (dark, low $\sigma$ ) activity of the network in the $(k, p)$ plane. The island that appears on the right side indicates that the SW (for some range of values of $k$ ) is the only regime capable to produce fast coherent oscillations in the average activity after the presentation of the stimulus. values at different periods of the global average activity. The ability to represent the coding observed in the AL [3] can be seen only in regular and SW networks. However, we remind the reader that regular networks have very slow activation times.

In conclusion, regular networks produce coherent oscillations in a slow time scale, whereas random networks give rise to fast response but without coherent oscillations. We have observed that SW networks show both coherent oscillations with the ability of temporal coding and fast reaction times. The dynamical system introduced in the nodes of the network is the Hodgkin-Huxley model that presents a saddle-node bifurcation to the limit cycle. Another research direction could analyze a different dynamical system with a different type of bifurcation in the node, such as the Hopf. It remains to be seen if the results presented in this paper can be extrapolated to other dynamical systems with different kinds of bifurcations.

Although we have not performed a detailed analysis of the mechanism that generates coherence, the simulations show that (i) it takes longer to synchronize in regular networks because the localized input needs to propagate through the ring, (ii) the SW topology overcomes this problem because of the existence of a few long range connections, and (iii) in the random case the clustering coefficient is too low, which implies that a specific neuron
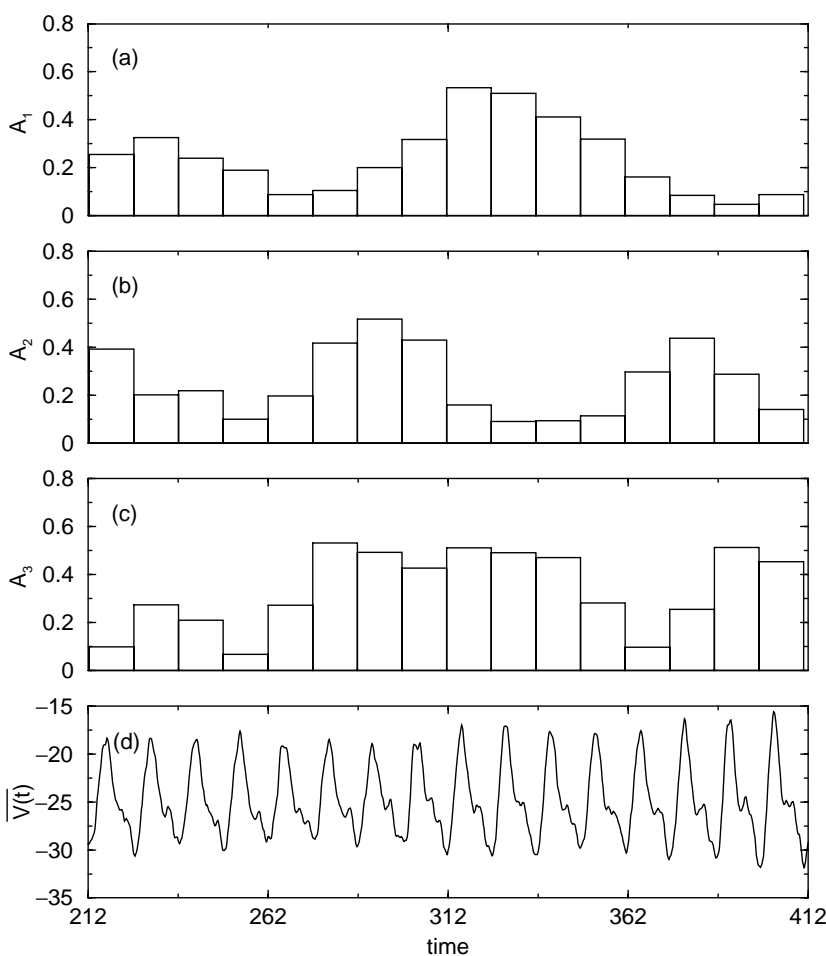

FIG. 4. (a)-(c) Average activity of three different clusters of neurons averaged over periods of the global mean activity. The simulation corresponds with that of Fig. 2b, which lies within the SW region. (d) Average activity of the whole network showing the coherent oscillations over which the activities of clusters are averaged. 
receives signals from many neurons that do not communicate among themselves. However, in the random network, coherent oscillations are possible provided its clustering coefficient is sufficiently high. That is, if we increase the average number of connections per neuron $k$ in the random network we find a transition from nonoscillatory (low $k$ ) to oscillatory (high $k$ ). The sparse random connection case has been carefully studied by [16] using integrate-and-fire models. Our simulations are coherent with their results.

We acknowledge G. Laurent, A. Bäcker, M. Bazhenov, M. Rabinovich, and H. Abarbanel for insightful discussions. We thank the Dirección General de Enseñanza Superior e Investigación Científica for financial support (PB97-1448), the CAM for financial support to L.F.L., and the CCCFC (UAM) for the use of computation resources.

[1] D. J. Watts and S.H. Strogatz, Nature (London) 393, 440 (1998).

[2] A. Barrat and M. Weigt (to be published); J.B.M. Barthélémy and L. A. N. Amaral, Phys. Rev. Lett. 82, 3180 (1999); M. A. de Menezes, C. F. Moukarzel, and T. J. P. Penna (to be published); cond-mat/9903426; M. E. J. Newman and D. J. Watts, Phys. Lett. A 263, 341 (1999).

[3] M. Wehr and G. Laurent, Nature (London) 384, 162 (1996); G. Laurent and H. Davidowitz, Science 265, 1872 (1994);
K. MacLeod and G. Laurent, Science 265, 976 (1996); K. MacLeod, A. Bäcker, and G. Laurent, Nature (London) 395, 693 (1998).

[4] C. von der Marlsburg, in Models of Neural Networks II, edited by E. Domany, J. L. Van Hemmen, and K. Schulten (Springer-Verlag, Berlin, 1994).

[5] C. M. Gray, J. Comput. Neurosci. 1, 11 (1994).

[6] K. Kaneko, Physica (Amsterdam) 23D, 436 (1986).

[7] H. Chaté, A. Lemaitre, P. Marq, and P. Manneville, Physica (Amsterdam) 224A, 447 (1996).

[8] D. Hansel and H. Sompolinsky, Phys. Rev. Lett. 68, 718 (1992); C. van Vreeswijk and H. Sompolinsky, Science 274, 1724 (1996); I. S. Aranson, D. Golomb, and H. Sompolinsky, Phys. Rev. Lett. 68, 3495 (1992).

[9] C. Fohlmeister, W. Gerstner, R. Ritz, and J. L. van Hemmen, Neural Comput. 7, 1046 (1995).

[10] R. Huerta, M. Bazhenov, and M. I. Rabinovich, Europhys. Lett. 43, 719 (1998).

[11] A. L. Hodgkin and A. F. Huxley, J. Physiol. (London) 117, 500 (1952).

[12] S. G. Lee, A. Neiman, and S. Kim, Phys. Rev. E 57, 3292 (1999).

[13] T. E. Hull, W. H. Enright, B. F. Fellen, and R. E. Sedgwick, SIAM J. Num. Anal. 9, 603 (1972). The absolute error was $10^{-15}$ and the relative error was $10^{-7}$ in all the calculations presented in this Letter.

[14] A. Destexhe, Z.F. Mainen, and T. J. Sejnowski, Neural Comput. 6, 14 (1993).

[15] H. Gang, T. Ditzinger, C.Z. Ning, and H. Haken, Phys. Rev. Lett. 71, 807 (1993).

[16] D. Golomb and D. Hansel (to be published). 